\title{
Priority Based Weighted Queue QoS Model In MANETs
}

\author{
Rajwinder Kaur ${ }^{1}$, Ramnik Singh ${ }^{2}$ \\ ${ }^{I}$ (Student Of M.Tech Ece, Daviet/ Ptu, India) \\ ${ }^{2}$ (Asst.Prof In Ece, Daviet/ Ptu, India)
}

\begin{abstract}
Mobile ad-hoc networks (MANETs) is an appealing technology that has attracted lots of research efforts over past years. Although the principle of wireless, structure-less, dynamic networks is attractive, there are still some major flaws that prevent commercial expansion. QoS is one the main issue in todays multimedia technology. In this paper we purposed a model i.e, Priority Based Weighted Queue QoS model in MANETs. Model is implemented in NS-2.34 and results are compared with other models.
\end{abstract}

Keywords: QoS,MANET, End to End delay,bandwidth,NS -2.34

\section{Introduction}

Mobile Ad hoc Networks is a collection of wireless mobile nodes, which are self-organizing, rapidly deployable and require no fixed infrastructure . They are composed of wireless mobile nodes (e.g. a router consisting of multiple hosts and equipped with wireless communication capability) that can be deployed anywhere, and must cooperate in order to dynamically establish communications using limited network management and administration [1]. Nodes in an ad hoc network may be highly mobile, or stationary, and may vary widely in terms of their capabilities and uses. They may operate autonomously or connected to the Internet. MANET incorporates routing functionality into mobile nodes so that they become capable of forwarding packets on behalf of other nodes and thus effectively become the infrastructure. Providing multiple routing paths between any source-destination pair of nodes has proved to be very useful in the context of wired networks [2]. The emergence of real-time applications and the widespread use of wireless and mobile devices have generated the need to provide Quality-Of-Service $(\mathrm{QoS})$ support in wireless and mobile networking environments. The effective utilization of the bandwidth for the multimedia applications with QoS is certainly one of the major challenge for the future generation of wireless networks. The transmission of sensitive applications requires a greater amount of bandwidth from the server to the client. This results in a large number of delays and throughput is also decreased. This article presents a resource allocation method for the sensitive applications using the Priority Based Weighted Queue QoS model in MANETs for reducing delays and provides proper utilization of bandwidth to the end users.

\section{Literature Review}

Here we present some of the existing work on the sensitive application in the MANETs. There are many traffic scheduling algorithm for wired network .But they cannot be directly applied to the wireless networks due to fundamental differences between them. An important difference between the wired networks and wireless networks is that the channel capacity will vary randomly over time and space and is asynchronous between the users. Firstcome-first-serve is a straightforward method to allocate the resource for the end-users. One of the drawbacks of this model is that it does not provide QoS for end users.

FCFS may be the simplest way for a scheduler to schedule the packets. In fact, FCFS does not consider the QoS parameters of each packets, it just sends the packets according to the order of their arrival time. Thus, the QoS guarantee provided by FCFS is in general weak and highly depends on the traffic characteristic of flows.[3]

(Shreedhar et al.), proposed a new approximation of fair queuing model called as deficit fair queue model. In this model the incoming packets are classified based on the destination address. It uses hash function to map incoming packets to the available queue. The different flow of the packets can reside in the same queue despite their importance. The hash function changes periodically in order to avoid the packets, which are coming from the same source to reside in the same queue.[4] (Mclaughlin et al), proposed a new approximation of fair queuing that we call Round Robin Method. It allocates the resource without knowing the mean packetsize in advance. It is only suitable for small networks Iin this method a deficit counter is used for the processing of data .This method has a drawback that it produces longer delay and takes more bandwidth for transmission. Thus, for more dynamic network, it does not ensure QoS to end user.[5] (Varaprasad et al.) proposed a weighted 
fair queue model for the resource allocation in manets.It is based upon logically allocating separate fraction of resources during bottleneck. It classified data into queue based upon source destination pair,ports, socket no, type of service value, It work as conserving model providing QoS for end users.It is priority based queuing method that reserves bandwidth before transmission. The model needs to be incorporated with admission control algorithm for efficient transmission.[6]

\section{A Proposed Method}

The Priority Based Weighted Queue QoS model provides a queue by dividing the resources of the traffic based on the weights of the packets. The Prioity Based Weighted Queue model uses the traffic priority management system. The packets are transmitted in a timely fashion. In this model Bandwidth is allocated on priority basis (highest weight).The advantage of this method is that there is no pre-reservation of bandwidth is required .Hence, division of bandwidth is carried out properly. Therefore there is no wastage of bandwidth and delays will be reduced .Fig. 1 shows the proposed model.

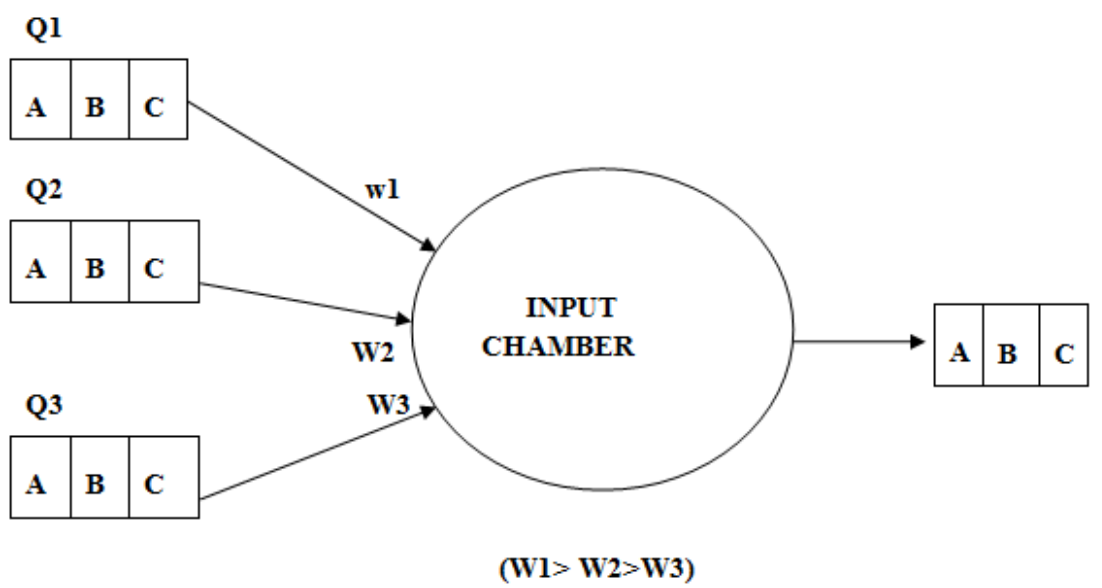

Figure 1. A Proposed Model

IV. Simulation And Results

Table 1: Simulation Parameters

\begin{tabular}{|l|l|}
\hline Topology size & $1500 \times 1500$ \\
\hline Simulation time & $2,000 \mathrm{~s}$ \\
\hline No.of users & 25 \\
\hline Mobility model & Random way point \\
\hline Pause time & $0 \mathrm{~s}$ \\
\hline Simulation & Ns -2.34 \\
\hline
\end{tabular}

This model considers an area of $1500 \mathrm{mX} 1500 \mathrm{~m}$ with a set of the mobile nodes placed randomly. The parameters are shown in Table1. The model is implemented using Network Simulator (NS-2.34). The simulation consists of 25 mobile nodes. Initially, each mobile node is placed at a random position within the defined area. 


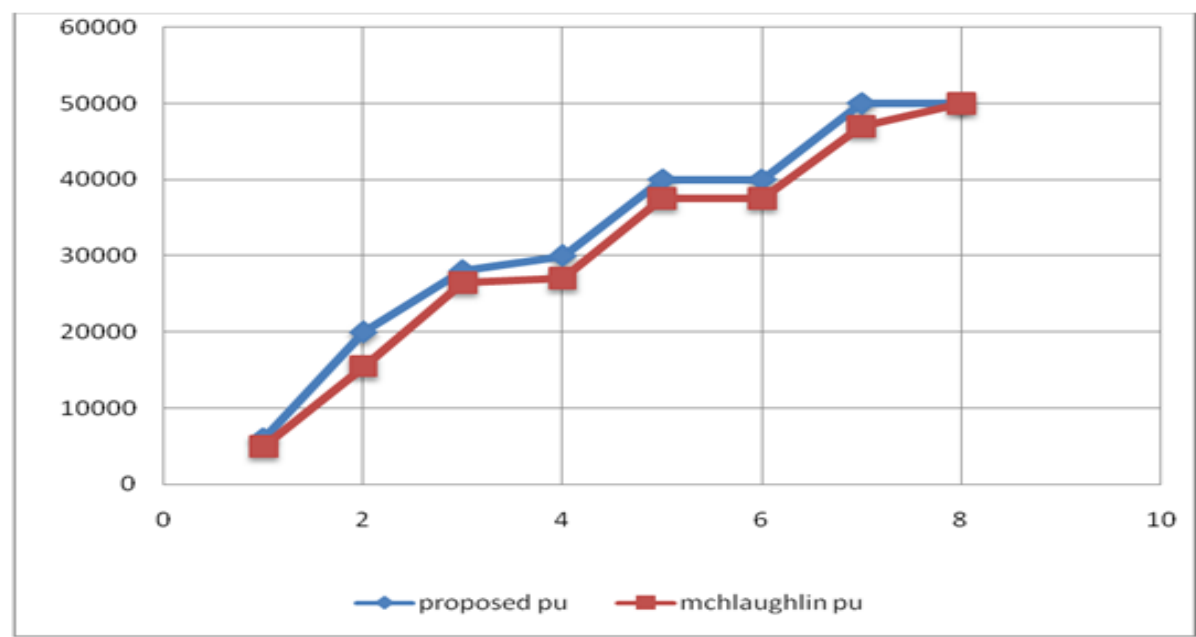

Figure 2. Previous vs Proposed PU

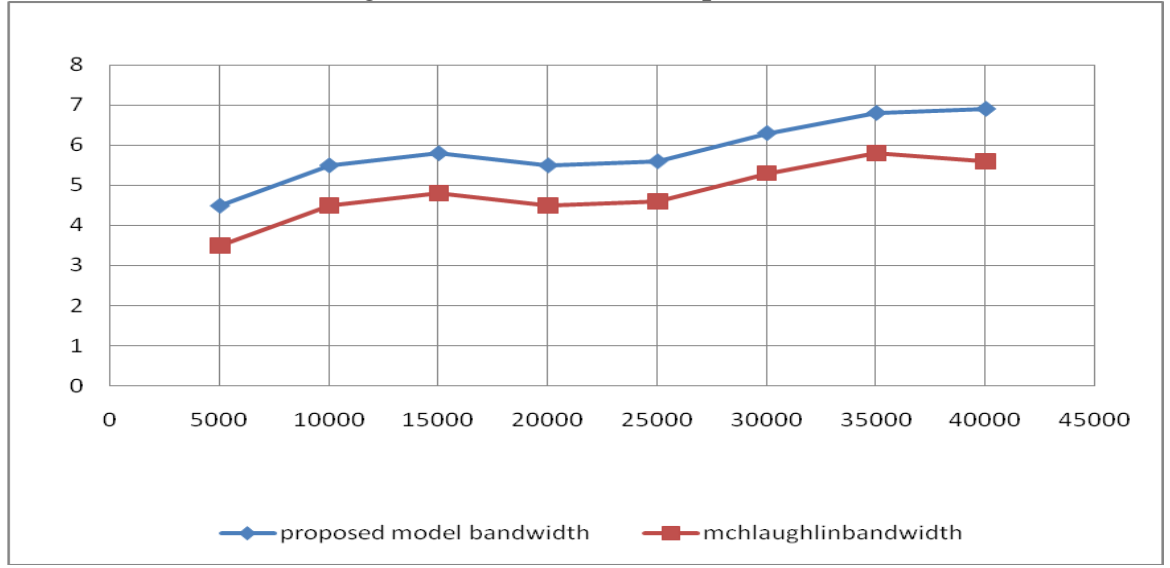

Figure 3.Previous vs Proposed Bandwidth

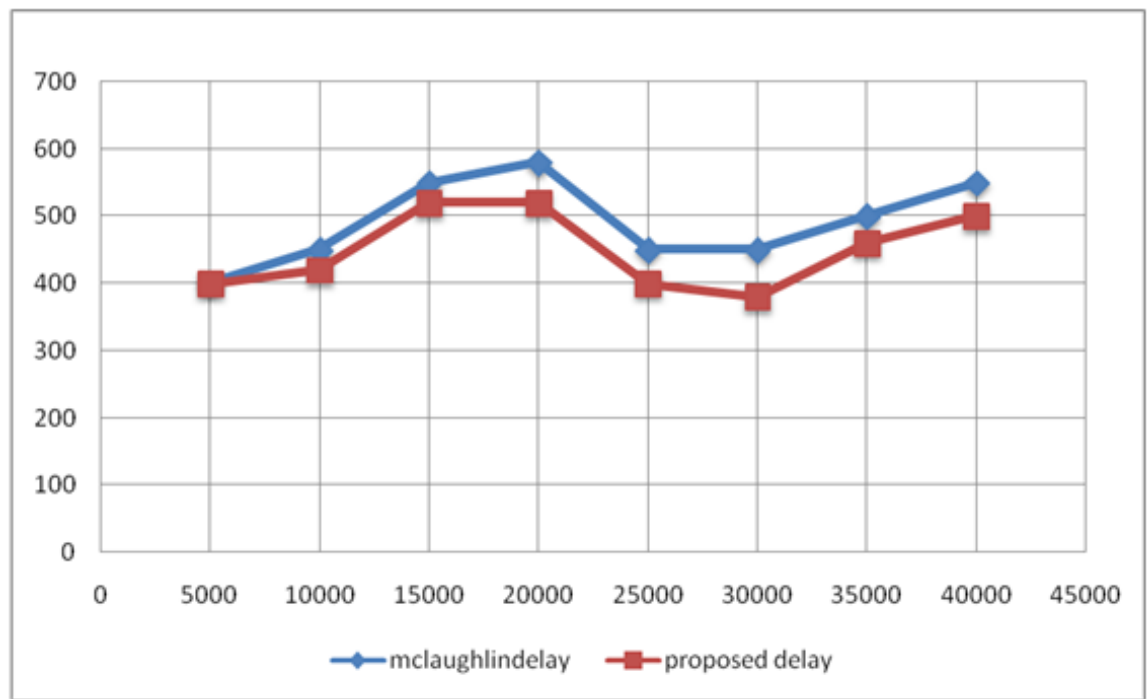

Figure.4: Previous vs Proposed End to End Delay

The proposed model has been compared with McLaughlin model. The number of PUs against the bandwidth is shown in Fig. 2. From the results, it is observed that as the number of the applications increases, the percentage of the bandwidth consumption also increases as compared to previous McLaughlin bandwidth. The number of PUs transferred in the proposed model is 40,000 wheras in McLaughlin PUs transferred is 37500. The number of PUs against bandwidth is shown in Fig. 3. The number of PUs in the proposed model is 30000 and the usage of the bandwidth is 6.3 Mbps compared to the McLaughlin model with $5.3 \mathrm{Mbs}$. Fig. 4 
illustrates the number of PUs versus end-toend-delay. In the proposed model, 5000PUs (arrival rate) take 390 ms to deliver from the source to the destination, whereas in the McLaughlin model it has taken $400 \mathrm{~ms}$. For 30000 PUs in the proposed model, it takes $380 \mathrm{~ms}$ as compared to $450 \mathrm{~ms}$ in the McLaughlin model.

\section{Conclusion}

We have discussed the resource allocation methods for MANETs. We propose a method to allocate the resources for the end-users in MANETs. The proposed algorithm has used a PRIORITY BASED WEIGHTED QUEUE QoS model to allocate the bandwidth and stream synchronization. It has been tested to evaluate the network performance using NS-2.34. The simulation results have shown that the packets are transmitted with the minimum delay at the high load. The proposed model takes only $400 \mathrm{~ms}$ for transmission of 30,000 PUs from the source to the destination and more number of presentation units PUs are transmitted with less utilization of bandwidth.

\section{References}

[1] Neeraj Nehra, R.B. Patel, V.K. Bhat, "Routing with Load Balancing in Ad Hoc Network: A Mobile Agent Approch", 6th IEEE/ACIS International Conference on Computer and Information Science (ICIS 2007), 2007 IEEE

[2] M. Shreedhar and G. Varghese ,"Efficient fair queuing using deficit round robin, (1995)," IEEE/ACM Trans. Networking, vol. 4, no. 3, pp. 375-385.

[3] Tsung-Yu Tsai et .al, "Introduction to Packet Scheduling Algorithms for Communication Networks," Institute for Information Industry Graduate Institute of Communication Engineering."

[4] K . McLaughlin, S. Sezer, H. Blume, X. Yang, F. Kupzog, and T. Noll ,"A Scalable Packet Sorting Circuit for High-Speed (7 july 2008)," IEEE Trans. On very large scale integration (VLSI) system, vol. 16, no. 7 .G.

[5] Varaprasad, R.S.D. Wahidabanu , "Quality of Service Model for Multimedia Applications in a Mobile Ad Hoc Network,(2011)," IEEE Potentials, vol. 30, no. 2, pp. 44-47.

[6] Maysam Hedayati, Hamid reza hoseiny, Seyed Hossein Kamali, Reza Shakerian, “ Traffic Load Estimation and Load Balancing in Multiple Routing Mobile Ad Hoc Networks", 2010 International Conference on Mechanical and Electrical Technology(ICMET 2010), 2010 IEEE, pp 117-121

[7] P. Goudarzi, M. Hosseinpourr, "Quality of Service Model for Multimedia Applications in a Mobile Ad Network,(2010),Consumer Electronics, IEEE Transactions on, vol. 56, no. 4, pp. 2217-2225.

[8] www.isi.edu/nsnam/ns/ns-documentation. 